\title{
ON THE RELIABILITY OF STELLAR AGES AND AGE SPREADS INFERRED FROM PRE-MAIN-SEQUENCE EVOLUTIONARY MODELS
}

\author{
Takashi Hosokawa ${ }^{1,2}$, Stella S. R. OfFner ${ }^{3}$, and Mark R. Krumholz ${ }^{4}$ \\ ${ }^{1}$ Jet Propulsion Laboratory, California Institute of Technology, Pasadena, CA 91109, USA; Takashi.Hosokawa@jpl.nasa.gov, hosokwtk@gmail.com \\ ${ }^{2}$ Department of Physics, Kyoto University, Kyoto 606-8502, Japan \\ ${ }^{3}$ Harvard-Smithsonian Center for Astrophysics, 60 Garden Street, Cambridge, MA 02138, USA \\ ${ }^{4}$ Department of Astronomy and Astrophysics, University of California, Santa Cruz, CA, 95064, USA \\ Received 2011 January 18; accepted 2011 June 13; published 2011 August 19
}

\begin{abstract}
We revisit the problem of low-mass pre-main-sequence stellar evolution and its observational consequences for where stars fall on the Hertzsprung-Russell diagram (HRD). In contrast to most previous work, our models follow stars as they grow from small masses via accretion, and we perform a systematic study of how the stars' HRD evolution is influenced by their initial radius, by the radiative properties of the accretion flow, and by the accretion history, using both simple idealized accretion histories and histories taken from numerical simulations of star cluster formation. We compare our numerical results to both non-accreting isochrones and to the positions of observed stars in the HRD, with a goal of determining whether both the absolute ages and the age dispersions inferred from non-accreting isochrones are reliable. We show that non-accreting isochrones can sometimes overestimate stellar ages for more massive stars (those with effective temperatures above $\sim 3500 \mathrm{~K}$ ), thereby explaining why non-accreting isochrones often suggest a systematic age difference between more and less massive stars in the same cluster. However, we also find the only way to produce a similar overestimate for the ages of cooler stars is if these stars grow from $\sim 0.01 M_{\odot}$ seed protostars that are an order of magnitude smaller than predicted by current theoretical models, and if the size of the seed protostar correlates systematically with the final stellar mass at the end of accretion. We therefore conclude that, unless both of these conditions are met, inferred ages and age spreads for cool stars are reliable, at least to the extent that the observed bolometric luminosities and temperatures are accurate. Finally, we note that the time dependence of the mass accretion rate has remarkably little effect on low-mass stars' evolution on the HRD, and that such time dependence may be neglected for all stars except those with effective temperatures above $\sim 4000 \mathrm{~K}$.
\end{abstract}

Key words: accretion, accretion disks - Hertzsprung-Russell and C-M diagrams - stars: evolution - stars: formation - stars: low-mass - stars: pre-main sequence

Online-only material: color figures

\section{INTRODUCTION}

Pre-main-sequence (PMS) stars in low-mass star-forming regions show a sizable luminosity spread when placed on the Hertzsprung-Russell diagram (HRD; e.g., Hillenbrand 2009). This spread translates into a significant dispersion in inferred stellar ages that records the past star formation activity in each region (e.g., D'Antona \& Mazzitelli 1994; Baraffe et al. 1998; Siess et al. 2000; Palla \& Stahler 2000, 1999; Hartmann 2001, 2003). However, the idea that star clusters form over an extended period is subject to extensive debate on both observational and theoretical grounds (e.g., Elmegreen 2000; Hartmann et al. 2001; Tan et al. 2006; Krumholz \& Tan 2007; Evans et al. 2009), and several authors have claimed that the dispersion of stellar luminosities does not reflect a real age spread. Members of young binaries and multiples exhibit a tighter age correlation, supporting the existence of an intrinsic age distribution. However, a luminosity spread persists even among such systems, and some companions display a substantial age mismatch (Prato et al. 2003; Stassun et al. 2008; Kraus \& Hillenbrand 2009). Deriving stellar ages is complicated from an observational standpoint. Calculation of stellar bolometric luminosities is beset by uncertainties in extinction, photometric variability, and unresolved multiplicity. Calibration between the stellar spectral type and effective temperature is also not trivial. In some cases, it can be demonstrated that observational uncertainties alone are sufficient to induce an age spread of $>10 \mathrm{Myr}$ and mask a coeval stellar population (Slesnick et al. 2008). However, Da Rio et al. (2010a; 2010b) carefully model these uncertainties and conclude that these effects alone cannot reproduce the entire spread. Other age indicators such as stellar rotation rate (Littlefair et al. 2010), surface gravity (Slesnick et al. 2008), and lithium abundances (Sestito et al. 2008) also support the idea that the inferred age spreads are real, but are each subject to significant challenges.

Apart from the observational uncertainties, physical mechanisms may be responsible for a portion of the observed HRD scatter. For the purpose of inferring stellar ages, it is usually assumed that PMS stars first appear along a "birth line" in the HRD when mass accretion ceases (e.g., Palla \& Stahler 1990; Hartmann et al. 1997). However, luminosities of younger embedded stars (Class 0 and I sources) that are presumably still accreting also show a wide spread, and a fraction of them have luminosities much lower than the values expected from the standard birth line (e.g., Kenyon et al. 1990; Evans et al. 2009; Enoch et al. 2009). A solution for this "luminosity problem" is the scenario that mass accretion takes place very time dependently, repeating burst-like accretion phases and quiescent phases. Recent numerical simulations suggest that such episodic mass accretion is caused by gravitational fragmentation of a circumstellar disk (e.g., Vorobyov \& Basu 2005; Machida et al. 2011), though radiative warming from protostars alleviates it 
(e.g., Offner et al. 2009). Regardless of the ultimate explanation for the luminosities of Class 0 and I sources, the existence of young stars that fall well below the putative birth line is strong evidence that we must extend our PMS evolution models to include the accretion phase.

Baraffe et al. (2009, hereafter BCG09) study protostellar evolution with various episodic mass accretion histories and examine the resultant spread of PMS stars in the HRD. They argue that PMS stars of the same mass and age show some scatter in the HRD owing to variation of the early evolution resulting from complex accretion histories. However, BCG09 simultaneously vary not only their accretion histories, but also their initial stellar models and the radiative properties of the accretion flow. Because they change these parameters in correlated ways and do not perform a systematic survey of parameter space, it is not clear which of these effects drives their results. Nor is it clear whether the results they generate via their parameter choices are consistent with observed HRDs of clusters. Consequently, it is still unclear how much vigorous time-dependent accretion histories influence protostellar evolution.

In this paper, we aim to resolve this question by performing a systematic study of how PMS evolutionary tracks change as we alter the accretion history, the initial models, and the thermal efficiencies of mass accretion. We perform a systematic survey of parameter space in order to understand how each of these factors affects protostellar evolution. This enables us to answer the question of whether variation in any of these quantities could produce the appearance of an age spread in a population that is actually coeval.

The structure of the paper is as follows. In Section 2, we briefly explain our numerical method for modeling protostellar evolution. Section 3 is the main part of the paper, where the numerical results are presented. First, we investigate how different accretion histories influence protostellar evolution in Section 3.1. We next investigate protostellar evolution with differing initial models in Section 3.2 and with differing thermal efficiencies in 3.3. In Section 4, we combine all these results to draw general conclusions about the reliability of age and age spread estimates from PMS evolutionary tracks. Section 5 contains the summary and discussion.

\section{PROTOSTELLAR EVOLUTION MODELS}

We model protostellar evolution using the numerical code described by Hosokawa \& Omukai (2009) and Hosokawa et al. (2010). The code numerically solves the four stellar structure equations, taking into account mass accretion. For the following calculations, we adopt the OPAL opacity tables (Iglesias \& Rogers 1996) for high temperature $T>7000 \mathrm{~K}$, and other tables based on the work by Alexander \& Ferguson (1994) for the lower temperature. We employ mixing-length theory for heat transport in convective layers with a constant ratio of the mixing length to the pressure scale height of 1.5. We confirmed that our code reproduces the calculations by Stahler et al. (1980) and Palla \& Stahler $(1990,1992)$ in both the limits of hot spherical accretion and cold disk accretion, which we explain in more detail below (see Appendices in Hosokawa \& Omukai 2009 and Hosokawa et al. 2010).

We refer the reader to the Hosokawa et al. papers for full details of the numerical method, but one parameter is particularly important for the results of this paper. The thermal efficiency of mass accretion, i.e., the entropy carried into the star with accreting material, is a key parameter for protostellar evolution. Since a protostar grows by accretion, the average entropy in the stellar interior becomes higher with higher thermal efficiency. For a star with a fixed mass, the stellar radius is larger for higher interior entropy content. Thus, we naively expect that, even for fixed accretion history, protostars will have larger radii if the accretion flows onto them have higher thermal efficiencies.

Despite efforts in previous work, however, the concrete value of the thermal efficiency in low-mass star formation is not well constrained. Here we address this uncertainty by considering two limiting cases, representing schematic versions of two different accretion flow geometries: "hot" spherical accretion and "cold" disk accretion. In the hot accretion case, we envision that an accretion flow directly hits the stellar surface and forms an accretion shock front. The accretion flow may arrive in a disk, but in the hot case we imagine that the disk is thick enough so that the accretion column covers much of the stellar surface. As a result, a small fraction of the heat generated at the shock front is carried into the stellar interior. In this limit we solve for the steady structure of the gas accretion envelope as well as the stellar interior; the two are connected at the stellar surface with accretion shock jump conditions (e.g., Stahler et al. 1980; Hosokawa \& Omukai 2009). In contrast, in the cold accretion case we envision that accreting gas initially falls onto a circumstellar disk and then reaches the stellar surface via a thin accretion column connecting the disk and star. As a result, most of the stellar photosphere is not covered by an accretion column and is able to radiate freely. Accreting gas softly settles on the stellar surface, and when it is incorporated into the star it has the same entropy as gas in the stellar photosphere. In this case we do not solve for the structure of the accretion flow, and we instead adopt the ordinary photospheric boundary condition (e.g., Palla \& Stahler 1992; Hosokawa et al. 2010).

Our treatment of boundary conditions differs slightly from that of BCG09, who modeled the thermal efficiency with a parameter $\alpha$, the fraction of accreting internal energy absorbed by the star. However, our limiting cases of hot and cold accretion just correspond to their $\alpha=1$ and 0 cases, respectively. The only other difference between our and BCG09's method is that BCG09 assume instantaneous and uniform mixing of accreting material in the stellar interior (Siess \& Forestini 1996). In this case, the entropy of newly accreted material is assumed to be the same as the local values in the stellar interior, whereas in our cold case it is assumed to match the stellar photosphere. Note that, even in the hot case, only a small fraction of the accretion energy goes into heating the stellar matter. This is similar to the $\alpha=1$ case in BCG09, where the accretion energy acts as a uniform heating source that is distributed uniformly throughout the stellar interior (e.g., see Siess et al. 1997). As a result of this treatment, most of this energy escapes from the star without being absorbed by the stellar matter. Thus, the term "hot mass accretion," in both our treatment and BCG09's, indicates that mass accretion increases the average entropy in the stellar interior, not that the accretion flow is completely radiatively inefficient.

\section{RESULTS}

\subsection{Accretion History Variation}

First, we examine how variations in protostellar accretion histories affect stars' HRD evolution. To this end, we calculate a series of models, summarized in Table 1a. In order to isolate dependence on the accretion history from other effects, all these calculations use the same initial model and boundary conditions. 
Table 1

Model Parameters and Results

\begin{tabular}{|c|c|c|c|c|c|c|c|}
\hline Case & Accretion History & Boundary Condition & $R_{*, 0}\left(R_{\odot}\right)$ & $M_{*, d}\left(M_{\odot}\right)$ & $M_{*, f}\left(M_{\odot}\right)$ & $R_{*, f}\left(R_{\odot}\right)$ & $t_{f}(\mathrm{kyr})$ \\
\hline \multicolumn{8}{|c|}{ 1a. Fixed initial and boundary conditions, varying accretion history } \\
\hline $\mathrm{mC} 5-\mathrm{C}$ & $10^{-5} M_{\odot} \mathrm{yr}^{-1}$ & $\mathrm{C}$ & 1.5 & 0.074 & 0.9 & 1.3 & 90 \\
\hline $\mathrm{mE}-\mathrm{C}$ & Episodic & $\mathrm{C}$ & 1.5 & 0.07 & 0.9 & 1.8 & 90 \\
\hline $\mathrm{mO}-\mathrm{C}$ & Simulation $^{\mathrm{a}}$ & $\mathrm{C}$ & 1.5 & 0.075 & 0.45 & 1.3 & 110 \\
\hline $\mathrm{mOx} 2-\mathrm{C}$ & Simulation $^{\mathrm{a}}$ & $\mathrm{C}$ & 1.5 & 0.077 & 0.9 & 1.1 & 110 \\
\hline $\mathrm{mOx} 0.5-\mathrm{C}$ & Simulation $^{\mathrm{a}}$ & $\mathrm{C}$ & 1.5 & 0.073 & 0.23 & 1.3 & 110 \\
\hline $\mathrm{mC} 4-\mathrm{C}$ & $10^{-4} M_{\odot} \mathrm{yr}^{-1}$ & $\mathrm{C}$ & 1.5 & 0.076 & 0.9 & 0.92 & 9 \\
\hline $\mathrm{mC6}-\mathrm{C}$ & $10^{-6} M_{\odot} \mathrm{yr}^{-1}$ & $\mathrm{C}$ & 1.5 & 0.1 & 0.9 & 1.4 & 900 \\
\hline \multicolumn{8}{|c|}{ 1b. Fixed accretion history and boundary conditions, varying initial conditions } \\
\hline mC5-C-Ri-8 & $10^{-5} M_{\odot} \mathrm{yr}^{-1}$ & $\mathrm{C}$ & 8.0 & 0.093 & 0.9 & 1.8 & 90 \\
\hline mC5-C-Ri3.7 & $10^{-5} M_{\odot} \mathrm{yr}^{-1}$ & $\mathrm{C}$ & 3.7 & 0.09 & 0.9 & 1.7 & 90 \\
\hline $\mathrm{mC5}-\mathrm{C}$ & $10^{-5} M_{\odot} \mathrm{yr}^{-1}$ & $\mathrm{C}$ & 1.5 & 0.074 & 0.9 & 1.3 & 90 \\
\hline mC5-C-Ri1 & $10^{-5} M_{\odot} \mathrm{yr}^{-1}$ & $\mathrm{C}$ & 1.0 & 0.06 & 0.9 & 1.1 & 90 \\
\hline mC5-C-Ri0.65 & $10^{-5} M_{\odot} \mathrm{yr}^{-1}$ & $\mathrm{C}$ & 0.65 & 0.049 & 0.9 & 0.85 & 90 \\
\hline mC5-C-Ri0.3 & $10^{-5} M_{\odot} \mathrm{yr}^{-1}$ & $\mathrm{C}$ & 0.3 & 0.035 & 0.9 & 0.64 & 90 \\
\hline mC5-C-Ri0.25 & $10^{-5} M_{\odot} \mathrm{yr}^{-1}$ & $\mathrm{C}$ & 0.25 & 0.033 & 0.9 & 0.4 & 90 \\
\hline mC5-C-Ri0.2 & $10^{-5} M_{\odot} \mathrm{yr}^{-1}$ & $\mathrm{C}$ & 0.2 & 0.033 & 0.9 & 0.29 & 90 \\
\hline \multicolumn{8}{|c|}{ 1c. Fixed accretion history and initial conditions, varying boundary conditions } \\
\hline mC5-C-Ri3.7 & $10^{-5} M_{\odot} \mathrm{yr}^{-1}$ & $\mathrm{C}$ & 3.7 & 0.09 & 0.9 & 1.7 & 90 \\
\hline $\mathrm{mC} 5-\mathrm{C}$ & $10^{-5} M_{\odot} \mathrm{yr}^{-1}$ & $\mathrm{C}$ & 1.5 & 0.074 & 0.9 & 1.3 & 90 \\
\hline $\mathrm{mC} 5-\mathrm{H}$ & $10^{-5} M_{\odot} \mathrm{yr}^{-1}$ & $\mathrm{H}$ & 3.7 & 0.34 & 0.9 & 4.6 & 90 \\
\hline mC5-HC0.3 & $10^{-5} M_{\odot} \mathrm{yr}^{-1}$ & $\mathrm{H} \rightarrow \mathrm{C}\left(0.3 M_{\odot}\right)^{\mathrm{b}}$ & $3.7(3.0)^{\mathrm{b}}$ & 0.33 & 0.9 & 4.3 & 90 \\
\hline mC5-HC0.1 & $10^{-5} M_{\odot} \mathrm{yr}^{-1}$ & $\mathrm{H} \rightarrow \mathrm{C}\left(0.1 M_{\odot}\right)^{\mathrm{b}}$ & $3.7(2.6)^{\mathrm{b}}$ & 0.25 & 0.9 & 3.6 & 90 \\
\hline mC5-HC0.03 & $10^{-5} M_{\odot} \mathrm{yr}^{-1}$ & $\mathrm{H} \rightarrow \mathrm{C}\left(0.03 M_{\odot}\right)^{\mathrm{b}}$ & $3.7(3.2)^{b}$ & 0.17 & 0.9 & 2.9 & 90 \\
\hline
\end{tabular}

Notes.

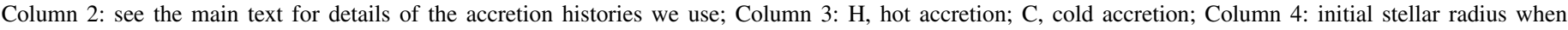

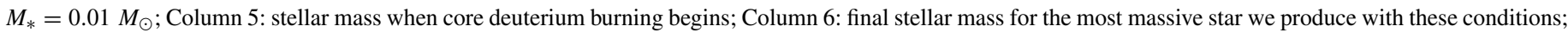

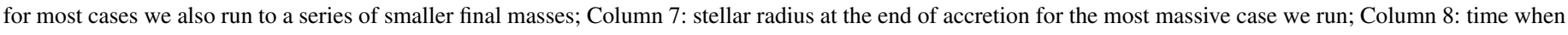
accretion ends for the most massive case we run.

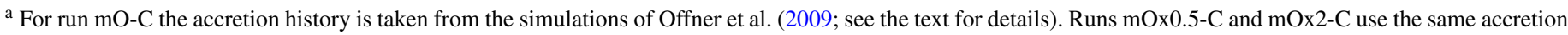
history, scaled by factors of 0.5 and 2 , respectively.

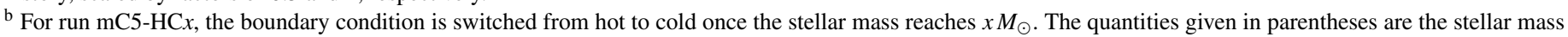
and radius when this switch occurs.

This initial model consists of a $0.01 M_{\odot}$ star with radiative interior, as in Stahler et al. (1980), and an initial radius of $1.5 R_{\odot}$. Note that the value of $1.5 R_{\odot}$ is a bit smaller than the radius of the seed protostar calculated by Masunaga \& Inutsuka (2000, hereafter MIO0), $4 R_{\odot}$. The boundary condition for all these models is cold accretion. We note that BCG09 obtained protostellar evolutionary tracks that deviate from the non-accreting isochrones substantially only for their cold cases (their $\alpha=0$ ), and this motivates us to focus on cold accretion first.

\subsubsection{Episodic Accretion}

We first consider five distinct accretion histories with varying degrees of episodic variation, but with only factor of $\sim 2$ level changes in the average accretion rate. This enables us to explore the sensitivity of stars' HRD evolution to the level of variability in their accretion histories. Model $\mathrm{mO}-\mathrm{C}$ uses an accretion history taken from numerical simulations of lowmass star formation by Offner et al. (2009) and is illustrated in Figure 1. In this case, the accretion rate gradually decreases over $\simeq 0.1 \mathrm{Myr}$, and the stellar mass finally reaches $0.45 M_{\odot}$. Models mOx0.5-C and mOx2-C use the same accretion history, but they are scaled by factors of 0.5 and 2 , respectively, to give final masses of $0.23 M_{\odot}$ and $0.9 M_{\odot}$. In contrast, model mC5-C uses a fixed accretion rate of $\dot{M}=10^{-5} M_{\odot} \mathrm{yr}^{-1}$. We

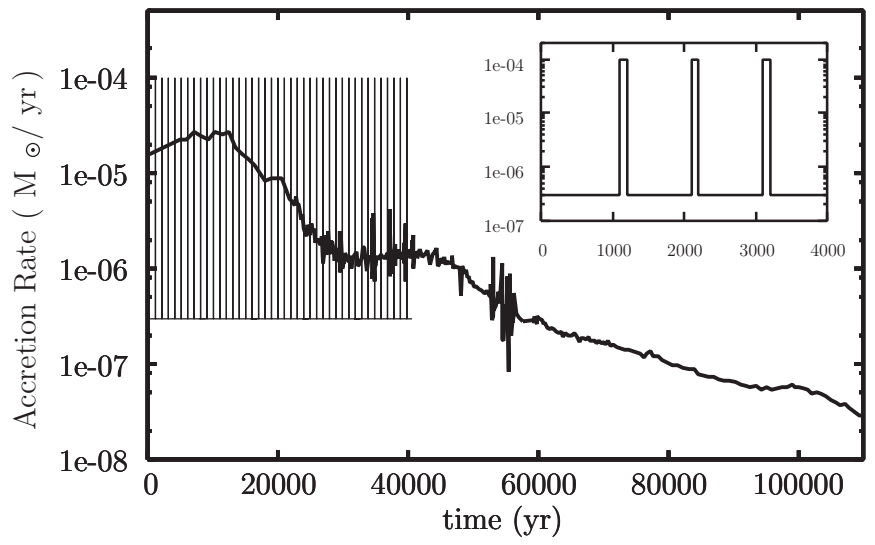

Figure 1. Time-dependent accretion histories adopted for calculations of protostellar evolution. The thick solid line presents the sample accretion history taken from the numerical simulations of low-mass star formation by Offner et al. (2009) and used in model mO-C. The thin solid line shows the vigorous episodic accretion case used in model $\mathrm{mE}-\mathrm{C}$, where a burst-like accretion phase at $10^{-4} M_{\odot} \mathrm{yr}^{-1}$ over 100 years is interspersed with a quiescent phase at $3 \times 10^{-7} M_{\odot} \mathrm{yr}^{-1}$ over 1000 years. The accretion history over initial 40,000 years is shown for this case. The small window enlarges the evolution over the initial 4000 years.

use this model to produce $0.23,0.45$, and $0.9 M_{\odot}$ stars, as in the mO-C models, simply by stopping accretion once the 


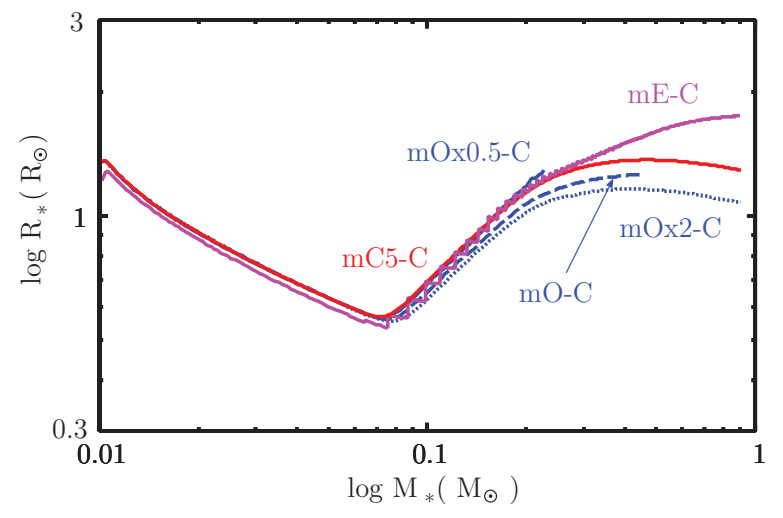

Figure 2. Evolution of the stellar radius vs. stellar mass for cases mC5-C (red), mE-C (magenta), mOx0.5-C (blue solid), mO-C (blue dashed), and mOx2-C (blue dotted), among which the accretion histories differ but the initial and boundary conditions do not.

(A color version of this figure is available in the online journal.)

star has reached the desired mass. Finally, model mE-C (also shown in Figure 1) represents an extreme case of variability: an episodic mass accretion history where burst-like accretion events at $\dot{M}=10^{-4} M_{\odot} \mathrm{yr}^{-1}$ over 100 years are interspersed with quiescent phases of accretion at $3 \times 10^{-7} M_{\odot} \mathrm{yr}^{-1}$ over 1000 years. This model is similar to the episodic accretion histories formulated by BCG09. As with mC5-C, we use this model to produce $0.23 M_{\odot}, 0.45 M_{\odot}$, and $0.9 M_{\odot}$ stars simply by turning off mass accretion once the stellar mass reaches the target value.

Figure 2 presents the evolution of the stellar radius until mass accretion ceases in each case. We see that the basic evolution is similar for all cases. The stellar radius initially decreases with increasing stellar mass. The temperature in the stellar interior rises during this initial contraction. When the stellar mass reaches $M_{*} \simeq 0.07 M_{\odot}$, deuterium burning begins and the stellar interior becomes fully convective. For some time after the ignition of deuterium, temperature at the stellar center remains constant at $\simeq 10^{6} \mathrm{~K}$ due to the very strong temperature dependence of the deuterium burning rate. This is the so-called thermostat effect of deuterium burning (e.g., Stahler 1988). The stellar radius increases in proportion to the stellar mass during this phase. The deuterium concentration in the stellar interior significantly decreases with increasing the stellar mass (Stahler 1988; Hartmann et al. 1997). Finally at $M_{*} \gtrsim 0.2 M_{\odot}$, the deuterium concentration is so low that the thermostat effect becomes inoperative and the central temperature increases again. Variation of the accretion histories only slightly influences the evolution of the stellar radius.

Figure $3^{5}$ shows the stellar positions in the HRD at $t=$ $0.3 \mathrm{Myr}, 1 \mathrm{Myr}, 3 \mathrm{Myr}$, and $10 \mathrm{Myr}$ after mass accretion begins for each mass accretion history. The snapshot at $t=0.3 \mathrm{Myr}$ shows the stellar positions just after mass accretion ceases. Reflecting the minimal variation in the stars' radii shown in Figure 2, the stellar positions only show a small spread. This means that the concept of the birth line is valid even with variable accretion histories in the limiting case of cold mass accretion, provided the initial state is the same from star to star.

\footnotetext{
5 Note that this figure is intended to facilitate comparison between the models and data for PMS stars, for which mass accretion has presumably ceased. In this and all subsequent figures we omit the accretion luminosity for the early evolutionary tracks. If the accretion luminosity is added, the tracks shift upward in the HRD and no longer pass through the data.
}

At $t>0.3 \mathrm{Myr}$, the stars gradually approach the zero-age mainsequence (ZAMS) line, descending in the HRD (Henyey et al. 1955; Hayashi 1961; Hayashi \& Nakano 1963). The snapshot at $t=1 \mathrm{Myr}$ clearly shows that the stars are below the $1 \mathrm{Myr}$ isochrone for non-accreting protostars. This offset is larger for the higher-mass stars. In particular, the $0.9 M_{\odot}$ stars are close to the isochrone of $10 \mathrm{Myr}$. The offset decreases with time, but still remains even at $t=10$ Myr for $0.9 M_{\odot}$ stars.

This divergence between the accreting evolutionary histories and the non-accreting isochrones is easy to understand. The isochrones for non-accreting protostars are derived assuming a large initial radius of $R_{*, 0} \sim 10 R_{\odot}$. The model PMS stars then contract from this initial state by radiating away their energy, reaching smaller radii at larger stellar ages. On the other hand, with thermally inefficient accretion the stellar radius remains as small as $R_{*} \lesssim 1 R_{\odot}$ during mass accretion. As a result, stellar ages are overestimated using the isochrones for non-accreting protostars in such cases. However, we stress that this effect has nothing to do with the time variability of the mass accretion rate.

\subsubsection{Varying Mean Accretion Rates}

Next we examine protostellar evolution over a greater range of mass accretion rates but with no time variability. In addition to case $\mathrm{mC5}-\mathrm{C}$, we consider cases $\mathrm{mC} 4-\mathrm{C}$ and $\mathrm{mC6}-\mathrm{C}$, which have constant accretion rates of $10^{-4} M_{\odot} \mathrm{yr}^{-1}$ and $10^{-6} M_{\odot} \mathrm{yr}^{-1}$, respectively. Figure 4 is the same as Figure 3 but for these cases. We see larger variation of the tracks among these cases than in Figure 3, but the level of variation is still significantly smaller than the observed range of data, particularly for the lowest mass and effective temperature. There is little difference between cases $\mathrm{mC5}-\mathrm{C}$ and $\mathrm{mC6}-\mathrm{C}$, where accretion rates take typical values for low-mass star formation of $\dot{M} \lesssim 10^{-5} M_{\odot} \mathrm{yr}^{-1}$. The mC4-C track slightly deviates from these tracks. In this case, however, the $0.9 M_{\odot}$ star always lies below the $10 \mathrm{Myr}$ isochrone, far from the locations where observed protostars lie. We therefore conclude that purely cold accretion at rates of $10^{-4} M_{\odot} \mathrm{yr}^{-1}$, while not physically forbidden, does not appear to actually occur in observed star clusters, at least for $0.9 M_{\odot}$ stars. The problem that many evolutionary scenarios involving cold accretion overpopulate the region at low $L$ and high $T_{\text {eff }}$ is one we will encounter repeatedly in the rest of this paper.

In summary, we conclude that star-to-star differences in either the overall accretion rate or the degree to which the accretion rate varies in time have a very limited effect on protostellar evolution. As illustrated by Figures 3 and 4, varying accretion histories can cause absolute age estimates to be wrong for more massive stars, but cannot explain the observed broad spread of PMS stars in the HRD. If PMS stars' initial state and accretion boundary conditions were fixed, then a coeval population would form a much tighter sequence in the HRD than what we actually observe, even if their accretion histories varied wildly.

\subsection{Initial Model Variation}

We next study protostellar evolution by varying the initial model, while fixing the boundary condition to cold mass accretion and the accretion rate to $\dot{M}=10^{-5} M_{\odot} \mathrm{yr}^{-1}$. We choose cold accretion here, because protostellar evolution is only sensitive to the initial model in the cold case, not the hot one (Stahler 1988; Hartmann et al. 1997)—we defer a detailed discussion of this issue to Section 3.3. Throughout these calculations, we assume a fixed initial deuterium abundance, which in principle may vary somewhat. Stahler (1988) explored the effect of varying deuterium abundance on the stellar radius. 

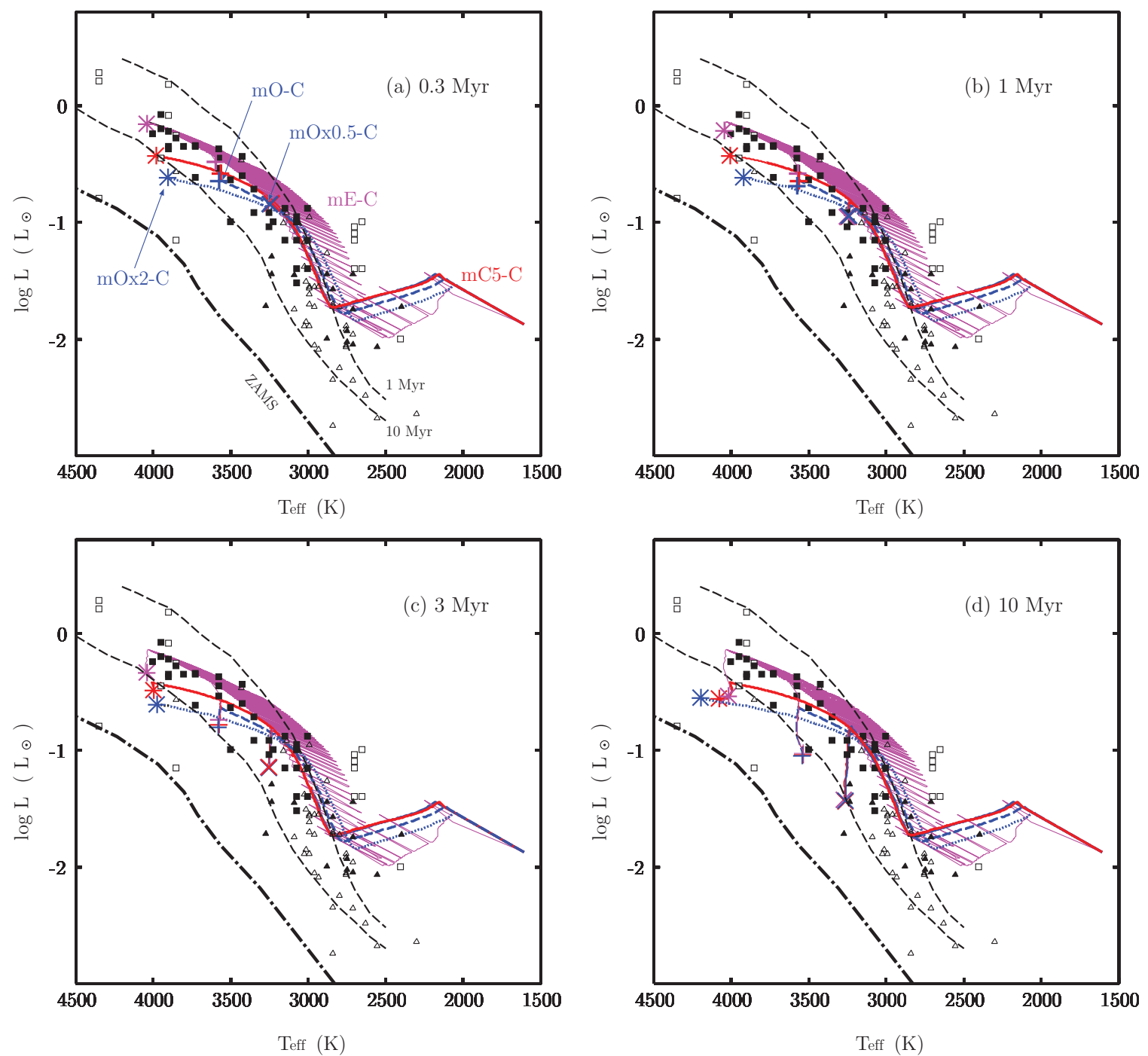

Figure 3. Snapshots of stellar positions in the HRD for varying accretion histories with the same initial and boundary conditions. The four panels show the snapshots at times $0.3 \mathrm{Myr}(\mathrm{a}), 1 \mathrm{Myr}$ (b), $3 \mathrm{Myr}$ (c), and $10 \mathrm{Myr}$ (d) after the start of mass accretion. The evolutionary tracks until that time are also plotted in the panels. (Note that the luminosity plotted here is only the stellar luminosity; accretion luminosity is not included.) The different colors represent different accretion histories: constant accretion at $10^{-5} M_{\odot} \mathrm{yr}^{-1}$ (red, case $\mathrm{mC5}-\mathrm{C}$ ), episodic accretion (magenta, case $\mathrm{mE}-\mathrm{C}$ ), and decreasing accretion (blue, cases mO-C, mOx2-C, mOx0.5-C). The input parameters in each case are summarized in Table 1a. In each panel, the symbols mark the positions of stars whose masses are $0.9 M_{\odot}$ (asterisks), $0.45 M_{\odot}$ (pluses), and $0.23 M_{\odot}$ (crosses). The thick dot-solid line indicates the positions of ZAMS stars (Siess et al. 2000). The dashed lines represent the isochrones of 1 Myr and $10 \mathrm{Myr}$ for non-accreting protostars (Baraffe et al. 1998). Observational data are taken from Gatti et al. (2006, open squares), Gatti et al. (2008, filled squares), Muzerolle et al. (2005, open triangles), and Peterson et al. (2008, filled triangles).

(A color version of this figure is available in the online journal.)

He showed that larger amounts of deuterium led to somewhat larger stars. However, this had only a small effect on the total thermal energy due to the thermostat effect of deuterium burning, which acts to regulate the central stellar temperature.

Unlike the accretion history, which is a macroscopic property that almost certainly varies from star to star, the initial radius is at least partly fixed by microphysics. The initial model should correspond to the "seed" protostar which forms as a result of second collapse induced by collisional dissociation of hydrogen molecules in a thermally supported first core (e.g., Larson 1969; Winkler \& Newman 1980; Masunaga et al. 1998; MI00). The entropy content of the resulting seed protostar, and thus its initial radius, is therefore at least partly set by the properties of the hydrogen molecule, in which case we would not expect large star-to-star variations. Nonetheless, models of second collapse have not extensively explored the influence of factors like rotation or magnetic fields. Thus, to be conservative we consider a factor of $\sim 100$ variation in possible initial radii, extending to values both larger and smaller than the radius of $4 R_{\odot}$ computed by MI00. The smallest radii we consider are much smaller than have been produced in any calculation of second collapse. We list the full set of models we examine in Table $1 \mathrm{~b}$.

Figure 5 presents the evolution of the stellar radius until mass accretion ceases in each case. We see that for all stellar masses these cases vary much more than those in Figure 2. With the smaller initial radius, the stellar radius is also smaller after the stellar mass increases by mass accretion. In case mC5-C-Ri0.3, for example, the temperature in the stellar interior is initially higher compared to case mC5-C. As a result, deuterium burning begins earlier at $M_{*} \simeq 0.03 M_{\odot}$, and the stellar radius is always smaller than $1 R_{\odot}$.

Figure 6 shows the evolutionary tracks in the HRD until the stellar mass reaches $0.9 M_{\odot}$. We see that tracks with small 

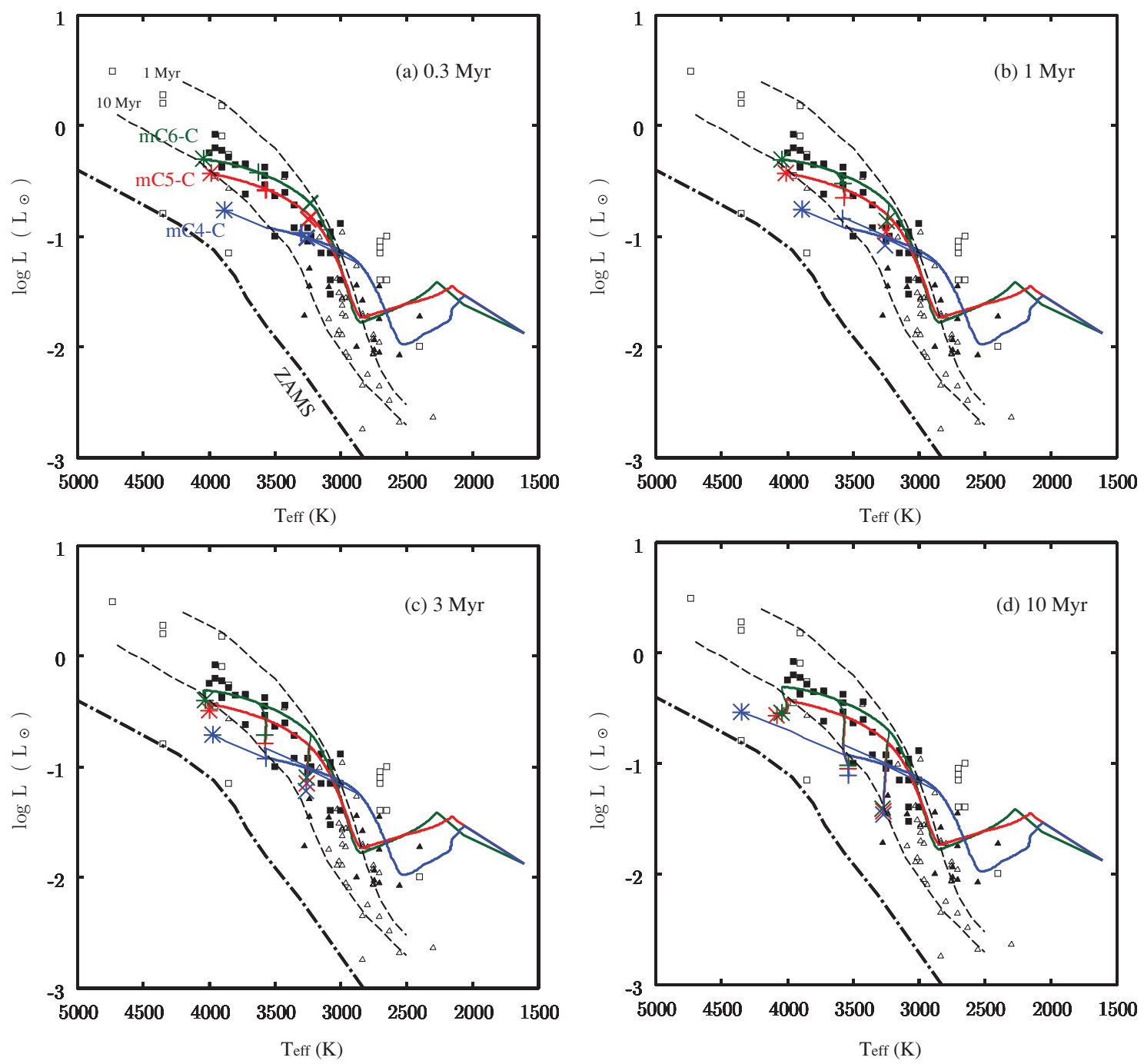

Figure 4. Same as Figure 3 but for different constant accretion rates. The different colors donate differences of the accretion rates: $10^{-6} M_{\odot} \mathrm{yr}^{-1}$ (green, case $\mathrm{mC6}-\mathrm{C}$ ), $10^{-5} M_{\odot} \mathrm{yr}^{-1}$ (red, case mC5-C), and $10^{-4} M_{\odot} \mathrm{yr}^{-1}$ (blue, case mC4-C). Parameters in each case are summarized in Table 1a.

(A color version of this figure is available in the online journal.)

initial radii occupy the lower part of the HRD, reflecting the variation shown in Figure 5. Figure 7 shows the evolution of the stellar positions in the HRD after mass accretion ceases for these cases, computed for stars that stop accreting at final masses of $0.05 M_{\odot}, 0.1 M_{\odot}, 0.3 M_{\odot}, 0.5 M_{\odot}, 0.7 M_{\odot}$, and $0.9 M_{\odot}$. In comparison to Figures 3 and 4, we see a much larger spread between the isochrones than that produced by different accretion histories. The stellar positions significantly differ even for the same mass and age. We see that the observational data points near the $10 \mathrm{Myr}$ non-accreting isochrone are covered even in the snapshots for $t \leqslant 1$ Myr.

However, with the exception of models mC5-C-Ri3.7 and mC5-C-Ri8, which start with large initial radii and entropies, the distribution of the calculated PMS stars is never consistent with that of the observational data points. Even at the earliest time snapshot, in these models most or all the stars with $T_{\text {eff }}>3500 \mathrm{~K}$ lie below the $10 \mathrm{Myr}$ isochrone, where there are no observed stars. This problem is particularly serious for the cases represented with blue symbols (cases mC5-C-Ri0.3, $\mathrm{mC} 5-\mathrm{C}-\mathrm{Ri} 0.25$, and $\mathrm{mC} 5-\mathrm{C}-\mathrm{Ri} 0.2$ ).

In order to render these cold accretion models with small radii consistent with observations, one would have to posit that only stars whose final masses are below $0.5 M_{\odot}$ have second cores with radii much smaller than the values predicted by MI00 and similar calculations. In effect, the $\sim 0.01 M_{\odot}$ second core would need to know in advance what the final properties of the star would be, and the properties of the second core would have to somehow correlate with the final mass. Given the limited range of second collapse models that have been explored in the literature, we cannot rule out the possibility that both very small second core radii and a systematic correlation between second core radii and final stellar masses exist in nature. However, we are also unaware of any observational or theoretical evidence in favor of either of these propositions.

If, on the other hand, we restrict our attention to models with initial radii such that the stars are at least marginally consistent with the data at all effective temperatures (models mC5-CRi-8 to mC5-CRi0.65, shown in green and red in Figure 7), we see that the spread in HRD location at $T_{\text {eff }}<3500 \mathrm{~K}$ is very small, comparable to the spread seen in Figures 3 and 4, and much smaller than the spread in observed stellar positions.

\subsection{Thermal Efficiency Variation}

Next, we consider the effects of different thermal efficiencies of mass accretion on protostellar evolution. In addition to the 


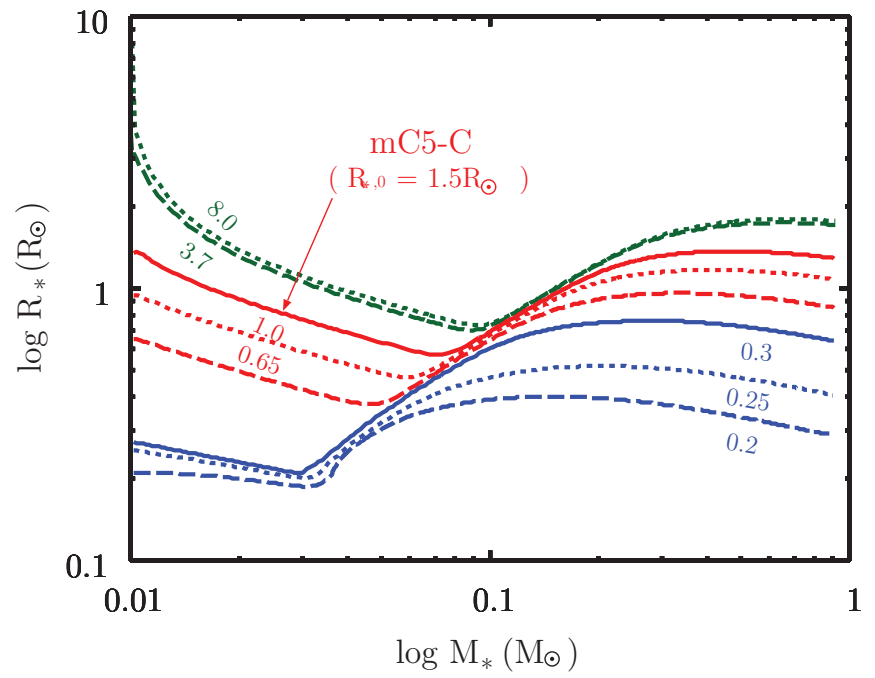

Figure 5. Same as Figure 2 but for cases with different initial radii: $R_{*, 0}=$ $8.0 R_{\odot}$ (green dotted, case mC5-C-Ri8), $R_{*, 0}=3.7 R_{\odot}$ (green dashed, case mC5-C-Ri3.7), $R_{*, 0}=1.5 R_{\odot}$ (red solid, case mC5-C), $1.0 R_{\odot}$ (red dotted, case mC5-C-Ri1), $0.65 R_{\odot}$ (red dashed, case mC5-C-Ri0.65), $0.3 R_{\odot}$ (blue solid, case mC5-C-Ri0.3), $0.25 R_{\odot}$ (blue dotted, case mC5-C-Ri0.25), and $0.2 R_{\odot}$ (blue dashed, case mC5-C-Ri0.2). The values of the initial radii are labeled in the panel. The cold mass accretion at the constant rate $\dot{M}=10^{-5} M_{\odot} \mathrm{yr}^{-1}$ is adopted for all the cases.

(A color version of this figure is available in the online journal.)

cold accretion models we have previously considered, we now add models that experience hot accretion for varying lengths of time during their evolution. We summarize these models in Table 1c. Model mC5-H uses pure hot accretion and models mC5-HC $x$ switch from hot to cold accretion once their masses exceed $x M_{\odot}$.

For these new hot models we adopt $0.01 M_{\odot}$ radiative stars for the initial state, as in the previous section. We use initial radii of $3.7 R_{\odot}$, which is roughly consistent with the value of $3.45 R_{\odot}$ in Stahler et al. (1980) and the value of $4.0 R_{\odot}$ computed by MI00, and somewhat larger than the value of $2 R_{\odot}$ adopted in Stahler (1988). ${ }^{6}$ However, the initial radius does not matter in the hot case as it does in the cold one (Stahler 1988; Hartmann et al. 1997). In the cold case, the entropy of gas accreting onto the star matches the entropy of the stellar atmosphere. Since the structure of the stellar atmosphere depends on the initial model, so does the subsequent evolution. In contrast, for hot models there is a self-regulation mechanism that removes the dependence on the initial radius. If the initial radius is too small, accreting gas releases a large amount of gravitational energy before reaching the stellar surface. Since a fraction of this energy is trapped in the accreting gas in the hot case, accreting materials settle onto the star with high entropy, which increases the stellar radius. On the other hand, if the initial radius is too large, the opposite effect operates. Accreting material has less entropy because it converts less of its gravitational energy to kinetic energy, and this serves to decrease the stellar radius. The stellar radius is regulated as a result.

Figure 8 shows the evolution of the stellar radius for the runs with varying thermal efficiency. In the cases with the early hot accretion, the stellar radius at $M_{*}=M_{*, \mathrm{HC}}$ is

\footnotetext{
6 The difference between Stahler et al. (1980) and Stahler (1988) arises because Stahler (1988) uses fully convective initial models, although the interior of a seed protostar would be radiative prior to deuterium burning (Stahler et al. 1980). In our calculations, we follow the early evolution, where a convective layer occurs after the ignition of deuterium burning.
}

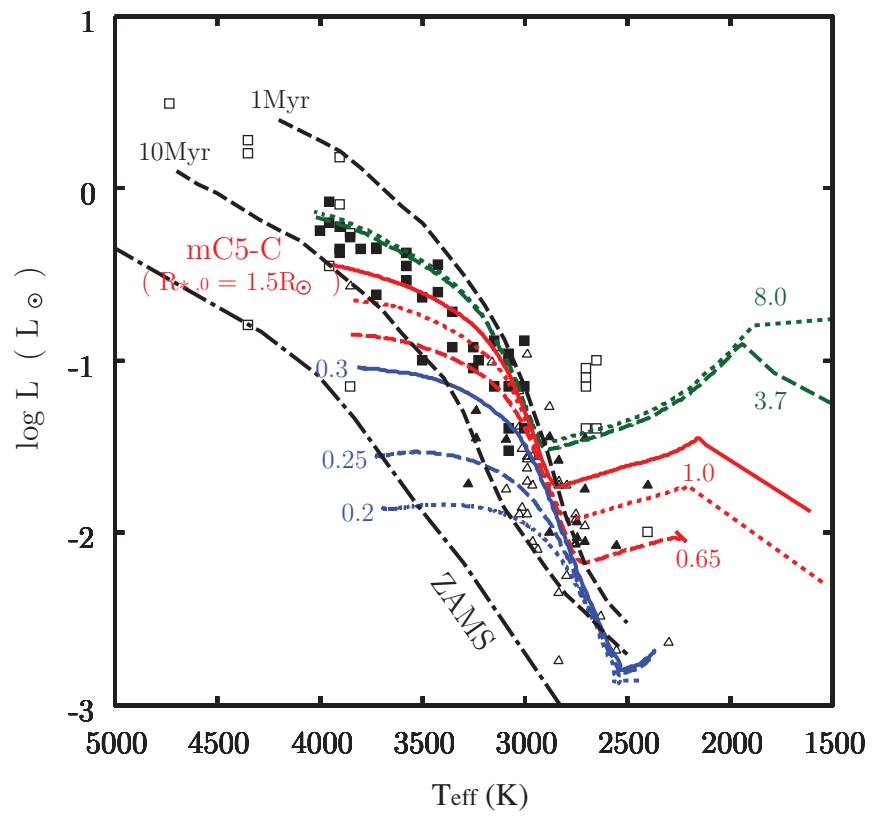

Figure 6. Evolution tracks with varying the initial radius with the cold mass accretion. The constant accretion rate of $10^{-5} M_{\odot} \mathrm{yr}^{-1}$ is adopted for these all cases. The tracks until the protostellar mass reaches $0.9 M_{\odot}$ by mass accretion are presented. The different lines show the evolution with different initial radii: $R_{*, 0}=8.0 R_{\odot}$ (green dotted, case mC5-C-Ri8), $R_{*, 0}=3.7 R_{\odot}$ (green dashed, case $\mathrm{mC5}-\mathrm{C}-\mathrm{Ri} 3.7), R_{*, 0}=1.5 R_{\odot}$ (red solid, case mC5-C), $1.0 R_{\odot}$ (red dotted, case mC5-C-Ri1), $0.65 R_{\odot}$ (red dashed, case mC5-C-Ri0.65), $0.3 R_{\odot}$ (blue solid, case mC5-C-Ri0.3), $0.25 R_{\odot}$ (blue dotted, case mC5-C-Ri0.25), and $0.2 R_{\odot}$ (blue dashed, case mC5-C-Ri0.2). The initial radius in each model is labeled in the figure. The input parameters for these models are summarized in Table $1 \mathrm{~b}$.

(A color version of this figure is available in the online journal.)

$\simeq 3 R_{\odot}$, significantly larger than in any of the purely cold cases considered in Section 3.2. In case mC5-HC0.1, for example, the stellar radius slightly decreases after the boundary condition is switched to cold mass accretion at $M_{*}=0.1 M_{\odot}$. However, deuterium burning begins soon after and the star expands for $M_{*} \gtrsim 0.3 M_{\odot}$. The evolution at $M_{*}>M_{*, \mathrm{HC}}$ is close to that in the purely hot case mC5-H. Even with our lowest switching mass $M_{*, \mathrm{HC}}=0.03 M_{\odot}($ case MC5-HC0.03), the stellar radius always exceeds $1 R_{\odot}$.

Figure 9 is the same as Figure 6 but for the cases with varying efficiency. We see that at the end of accretion all the models with any hot accretion, even $\mathrm{mC5}-\mathrm{HC} 0.03$ where we switch to cold accretion at $0.03 M_{\odot}$, lie at or above the 1 Myr nonaccreting isochrone and above the level of the observed data. The cold models are systematically lower. Significantly, only the hot models are consistent with the stars at the highest observed $L$ and $T_{\text {eff }}$. We therefore conclude that either these stars must experience some hot accretion, or that their initial radii must be very large, thereby achieving the same effect.

Figure 10 shows the subsequent time evolution of the stellar positions for these cases as in Figure 7. We omit cases mC5HC0.1 and mC5-HC0.3 here, because they are almost the same as case $\mathrm{mC5}-\mathrm{H}$. The snapshot at $t=0.3 \mathrm{Myr}$ shows that, in case $\mathrm{mC} 5-\mathrm{H}$, the stars are above the $1 \mathrm{Myr}$ isochrone when mass accretion ceases. The PMS stars descend in the HRD after this, and their positions are nearly consistent with the isochrones at $t \gtrsim 1 \mathrm{Myr}$. The differences in stellar positions between cases $\mathrm{mC} 5-\mathrm{H}$ and $\mathrm{mC5}-\mathrm{HC} 0.03$ are small even in the early snapshots when $t \leqslant 1 \mathrm{Myr}$, and at $T_{\text {eff }} \lesssim 3500 \mathrm{~K}$ the differences are particularly small. Thus, even a short duration 

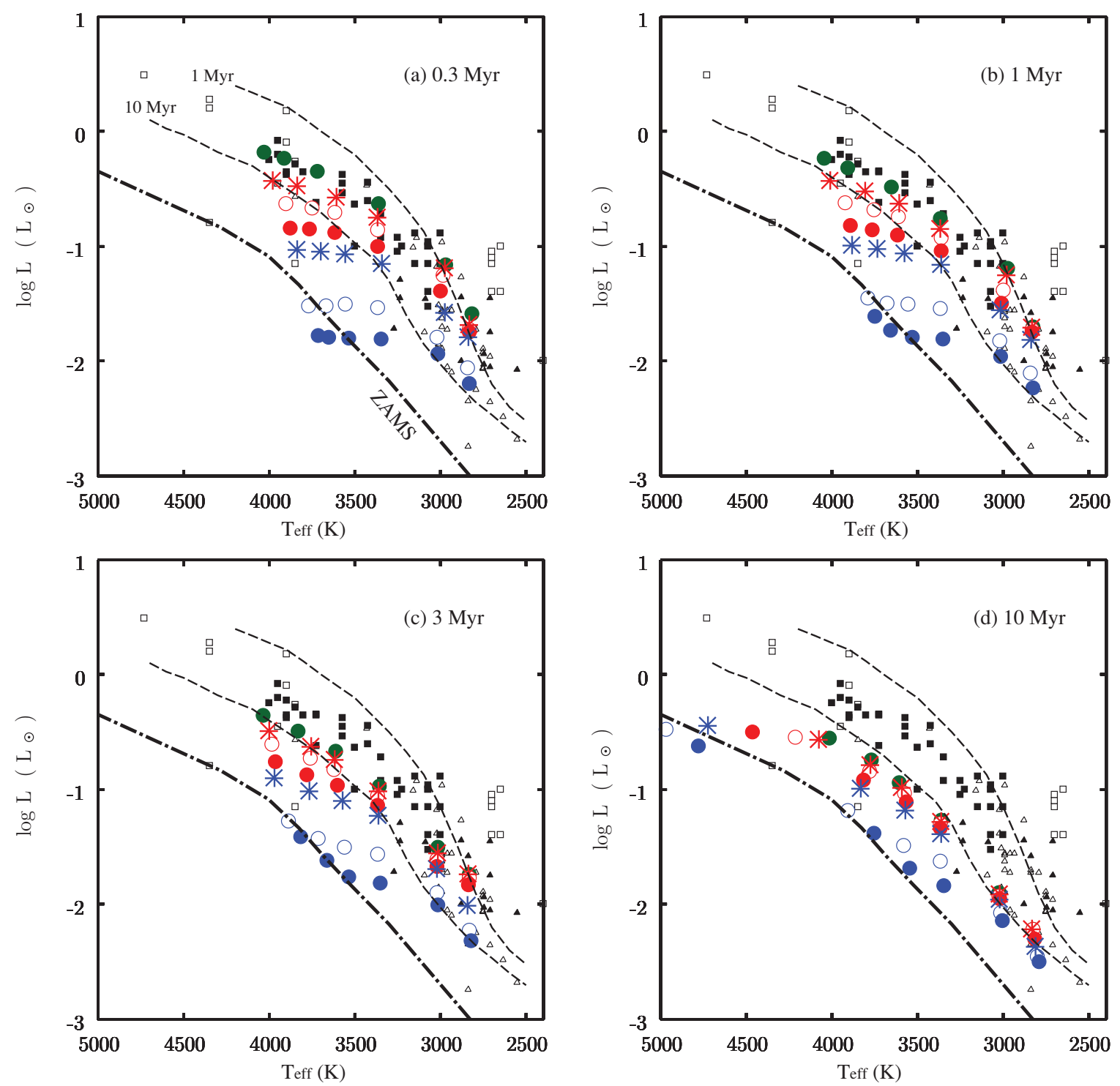

Figure 7. Same as Figure 3 but for the cases presented in Figure 6 and for the PMS stars of various masses. The symbols in each panel mark the positions of $0.9 M_{\odot}$, $0.7 M_{\odot}, 0.5 M_{\odot}, 0.3 M_{\odot}, 0.1 M_{\odot}$, and $0.05 M_{\odot}$ stars from the left for cases mC5-C-Ri3.7 (green filled circles), mC5-C (red asterisks), mC5-C-Ri1 (red open circles), mC5-C-Ri0.65 (red filled circles), mC5-C-Ri0.3 (blue asterisks), mC5-C-Ri0.25 (blue open circles), and mC5-C-Ri0.2 (blue filled circles). We omit the evolutionary tracks for clarity here. We also omit model mC5-C-Ri8 because it is nearly identical to mC5-C-Ri3.7.

(A color version of this figure is available in the online journal.)

of hot mass accretion produces a similar result to the purely hot case. BCG09 also find very bimodal outcomes between their hot cases $(\alpha \geqslant 0.2)$ and cold cases $(\alpha=0)$. This suggests that it is difficult to freely populate the PMS stars in the HRD only by varying the accretion thermal efficiency.

\section{THE RELIABILITY OF NON-ACCRETING ISOCHRONE ESTIMATES OF AGES AND AGE SPREADS}

Having explored the parameter space thoroughly, we are now in a position to make some general statements about the reliability of theoretical isochrones as tools for estimating ages and age spreads. In this analysis, it is helpful to separate the cases of stars with $T_{\text {eff }} \gtrsim 3500 \mathrm{~K}$ from those with $T_{\text {eff }} \lesssim 3500$, because the results are quite different in the two cases.

At $T_{\text {eff }}>3500 \mathrm{~K}$, stars with high and low thermal efficiencies, or with different initial radii, can end up quite far apart in the HRD even at equal ages (Figures 7 and 10). We therefore conclude that, in this regime, stellar age and mass alone cannot uniquely determine stellar positions in the HRD, and stellar age estimates based on HRD positions cannot in general be considered reliable. However, we note that errors in this regime only occur in one direction: young stars can appear old, but old stars never appear young. In no case do we find stars above the $1 \mathrm{Myr}$ isochrone whose ages are actually $>1$ Myr. Thus, very young age estimates are reliable in this effective temperature range, even if old age estimates are not. With regard to age spreads, we note that the snapshots at $t \lesssim 1$ Myr show that the models in which we vary the thermal efficiency and the initial radius cover the entire observed spread of PMS stars with $T_{\text {eff }}>3500 \mathrm{~K}$. Thus, the entire observed luminosity spread in this temperature range could be explained if a coeval population were to consist of some stars that underwent hot accretion and others that underwent cold accretion. Estimates of age spreads in this regime are therefore unreliable. 


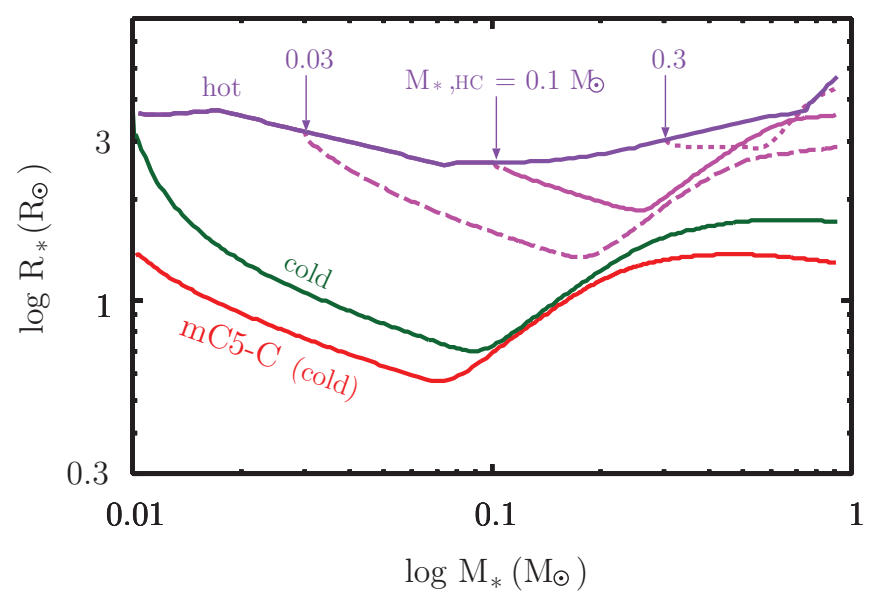

Figure 8. Same as Figure 2 but for cases with different thermal efficiencies of mass accretion. Only hot and cold mass accretion is adopted with the same initial radius $3.7 R_{\odot}$ in cases mC5-H (purple) and mC5-C-Ri3.7 (green). In the cases with the magenta lines, the hot mass accretion is switched to the cold accretion when the stellar mass reaches $M_{*, \mathrm{HC}}=0.3 M_{\odot}$ (magenta dotted, case $\mathrm{mC} 5-\mathrm{HC} 0.3$ ), $0.1 M_{\odot}$ (magenta solid, case mC5-HC0.1), and $0.03 M_{\odot}$ (magenta dashed, case mC5-HC0.03). The values of $M_{*, \mathrm{HC}}$ are labeled in the figure. The input parameters for these models are summarized in Table 1c. The red line represents the evolution in case $\mathrm{mC} 5-\mathrm{C}$ for a comparison.

(A color version of this figure is available in the online journal.)

The situation is quite different for $T_{\text {eff }} \lesssim 3500 \mathrm{~K}$. In this regime, consulting Figures 3, 4, 7, and 10 shows that the only models capable of producing false old ages are those that have pure cold accretion starting from very small initial radii. For example, even at a true age of $3 \mathrm{Myr}$, only model mC5-CRi0.2 places stars with $T_{\text {eff }}=3000 \mathrm{~K}$ below the $10 \mathrm{Myr}$ isochrone. However, these models can be considered acceptable and consistent with the observational data only if two uncertain propositions hold. First, a significant number of stars would have to accrete from seeds whose radii are an order of magnitude smaller than any that have been produced in theoretical models thus far. Second, in order to avoid drastically overpopulating the region below the $10 \mathrm{Myr}$ isochrone at $T_{\text {eff }} \gtrsim 3500 \mathrm{~K}$, such small initial radii would have to be realized only for stars that have small final masses at the end of accretion. There would have to be some mechanism to generate a correlation between the radii of $\sim 0.01 M_{\odot}$ second cores and the masses of the $\sim 1 M_{\odot}$ stars to which they eventually grow. Given the limitations of the theoretical models, neither of these propositions can be ruled out, but there is currently no evidence in favor of them either.

If we rule out the cold accretion, small initial radius models based on these problems, we find that the observed luminosity spread for $T_{\text {eff }}<3500 \mathrm{~K}$ is considerably larger than the spread among the remaining models. All these models lie fairly close to the non-accreting isochrones appropriate to their true ages for times $t \gtrsim 3 \mathrm{Myr}$ and at times $t \gtrsim 1 \mathrm{Myr}$ at $T_{\text {eff }} \sim 3000 \mathrm{~K}$. We therefore conclude that, unless $\sim 0.01 M_{\odot}$ seed protostars have some very specific unexpected properties, age estimates of 1-3 Myr or more based on non-accreting isochrones are reliable for stars with effective temperatures below $\sim 3500 \mathrm{~K}$, at least insofar as the observations themselves are reliable. Neither variation in thermal efficiency, accretion history, or initial radius can explain the observed spread in the HRD.

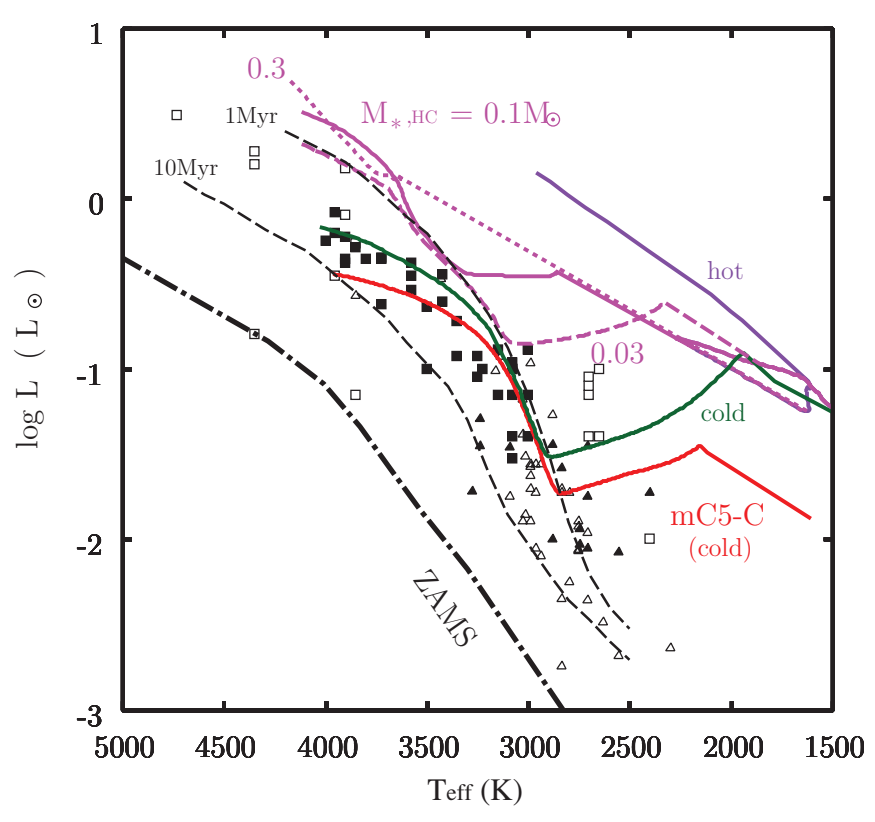

Figure 9. Same as Figure 6 but for varying the thermal efficiency of mass accretion. Different lines indicate the different thermal efficiencies: only hot mass accretion (purple, case $\mathrm{mC5}-\mathrm{H}$ ), cold accretion followed by early hot accretion for $M_{*}<M_{*, \mathrm{HC}}=0.3 M_{\odot}$ (magenta dotted, case mC5-HC0.3), $0.1 M_{\odot}$ (magenta solid, case mC5-HC0.1), $0.03 M_{\odot}$ (magenta dashed, case $\mathrm{mC} 5-\mathrm{HC} 0.03$ ), and only cold mass accretion with the same initial model as in the above cases (green, case mC5-C-Ri3.7).

(A color version of this figure is available in the online journal.)

\section{SUMMARY AND DISCUSSION}

In this paper, we have examined a variety of low-mass protostellar evolutionary tracks with varying accretion histories, initial models, and thermal efficiencies of mass accretion. We have also compared the resultant spread of PMS stars in the HRD to that observed in nearby low-mass star-forming regions (Peterson et al. 2008; Gatti et al. 2006; 2008; Muzerolle et al. 2005).

We first calculate protostellar evolution models with varying accretion histories but fixed initial stellar models and boundary conditions (Section 3.1). Our results show that if mass accretion is thermally inefficient, variation in the accretion history hardly influences protostellar evolution. Although isochrones for non-accreting protostars, such as those calculated by D'Antona \& Mazzitelli (1994), Baraffe et al. (1998) and Siess et al. (2000), do not necessarily provide us with correct stellar ages, models with differing accretion histories nevertheless form a tight sequence in the HRD. Thus, variable accretion histories alone cannot explain the observed spread of PMS stars in the HR. Moreover, the errors in absolute ages arise because non-accreting isochrones are not good descriptions of stars growing with thermally inefficient mass accretion. They are not a result of variable accretion histories. However, we note that this does suggest that accreting isochrones may resolve the problem of systematically larger inferred ages for high-mass cluster members (e.g., Hillenbrand 2009; Covey et al. 2010).

Second, we examine protostellar evolution with different initial models and thermal efficiencies of mass accretion, using a constant accretion rate $\dot{M}=10^{-5} M_{\odot} \mathrm{yr}^{-1}$ (Sections 3.2 and 3.3). We find that the spread of PMS stars in the HRD that results from varying the initial radius (or entropy) or the thermal efficiency is much larger than the spread that 

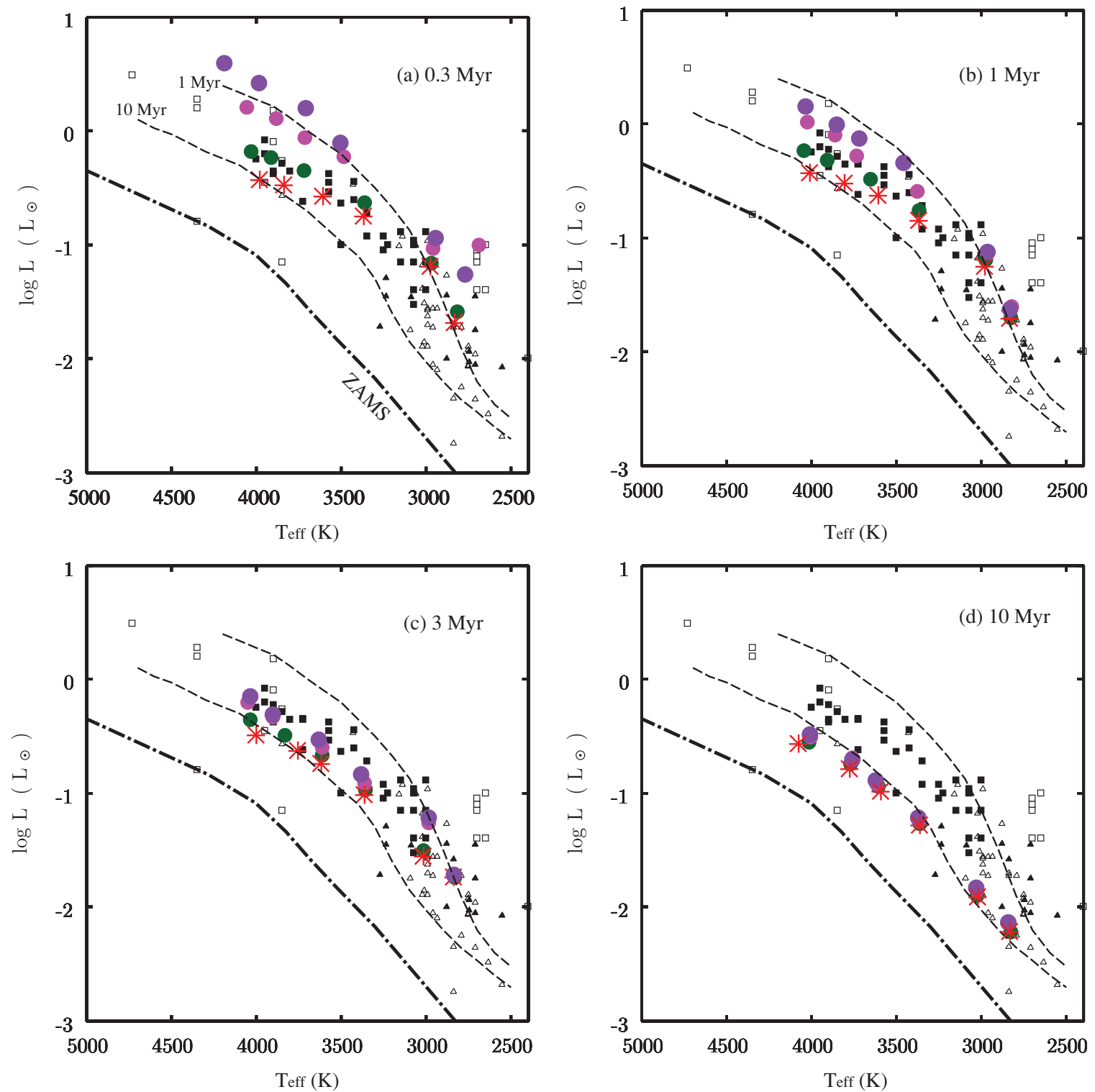

Figure 10. Same as Figure 7 but for the cases presented in Figure 9 . The symbols in each panel mark the positions of $0.9 M_{\odot}, 0.7 M_{\odot}, 0.5 M_{\odot}, 0.3 M_{\odot}, 0.1 M_{\odot}$, and $0.05 M_{\odot}$ stars from the left for cases mC5-H (purple filled circles), mC5-HC0.03 (magenta filled circles), mC5-C-Ri3.7 (green filled circles), and mC5-C (red asterisks).

(A color version of this figure is available in the online journal.)

arises from different accretion histories. We find that a coeval population of stars with significant star-to-star variation in thermal efficiency or initial radius could conceivably occupy the entire observed luminosity range for protostars with effective temperatures $\gtrsim 3500 \mathrm{~K}$. Thus, ages and age spreads observed in this temperature range may be unreliable. At lower effective temperature, however, the situation is very different. The only models we found in our parameter space survey that are capable of producing false old ages at low $T_{\text {eff }}$ are those with purely cold accretion starting from very small initial radii. However, we point out that these models require that $\sim 0.01 M_{\odot}$ second protostellar cores have radii $<0.3 R_{\odot}$, more than an order of magnitude smaller than any formed in simulations to date (e.g., MI00), and that they can be rendered consistent with observations only if such small initial radii are realized only for stars that end up growing to small final masses. Neither possibility can be definitively ruled out, but neither is supported by any current observations or theory either. If we exclude very small initial radii on these grounds, we find that all the remaining models indicate that ages and age spreads inferred from non-accreting isochrones are reliable for cool stars, at least to the extent that the observationally determined luminosities and temperatures are reliable (e.g., Da Rio et al. 2010a; 2010b).

In varying the thermal efficiency, we find that models with only a small amount of hot accretion, e.g., while $M_{*} \leqslant 0.03 M_{\odot}$, nonetheless show similar evolution to models with entirely hot accretion. Thus, in order to explain the HRD spread at low masses with cold accretion models, the models must begin with small radii and be thermally inefficient for nearly their entire evolution. Observational constraints on the thermal efficiency of accretion are somewhat tenuous. During the earliest stages during which accretion rates of $10^{-6}-10^{-4} M_{\odot} \mathrm{yr}^{-1}$ occur, protostars are too deeply embedded with too much 
radiation reprocessing to measure accretion signatures directly. Observations of $\mathrm{T}$ Tauri stars, for which accretion rates have declined to $\lesssim 10^{-7} M_{\odot} \mathrm{yr}^{-1}$, suggest that for these rates the accretion column covers only $\sim 1 \%-10 \%$ of the stellar surface. This supports a cold accretion scenario later in the accretion history. However, observations also find that the covering fraction increases with accretion rate (Gullbring et al. 2000; Ardila \& Basri 2000), suggesting that accretion may be more thermally inefficient at early times. Higher-mass stars, which experience higher initial accretion rates, seem unlikely to avoid hot accretion, while it is more observationally probable that very low mass stars experience purely cold accretion.

Our conclusions are different from those of BCG09, who stressed the significance of episodic mass accretion for explaining the observed spread of PMS stars in the HRD. However, our numerical results are actually consistent with theirs. First, BCG09 calculated protostellar evolution using simple, non-episodic accretion histories (their Figure 1) and found that, with thermally efficient accretion $(\alpha \geqslant 0.2$ in their notation), isochrones for non-accreting protostars give the correct ages at $t \gtrsim 1$ Myr. Disagreement with the isochrones arises only for thermally inefficient accretion flows $(\alpha=0)$. This is consistent with the results shown in our Figure 7 . Next, they calculated the evolution with more vigorous, episodic mass accretion histories (their Figure 2). However, the spread they obtain is no broader than that shown in their Figure 1, indicating that episodic accretion does not increase the HRD spread beyond what they had already introduced by using varying initial conditions and thermal efficiencies. Indeed, they state that the time dependence of the accretion rates is not essential for their results, and we confirm this finding. ${ }^{7}$ Our results suggest that BCG09 were able to obtain small luminosities for stars with $T_{\text {eff }} \lesssim 3500 \mathrm{~K}$, and thus claim to reproduce the observed HRD using a coeval population, because they used cold accretion starting from initial conditions with entropies far lower than what current theoretical models predict. They also did not continue runs with these initial conditions up to higher masses and effective temperatures. ${ }^{8}$ Our results suggest that, had they done so, the resulting stars would have fallen well below the locus of observed stars in the HRD, as do our comparable low initial entropy models.

Finally, we stress that we do not reject the episodic mass accretion as a possible solution for the "luminosity problem" of young embedded sources (Dunham et al. 2010; McKee \& Offner 2010; Offner \& McKee 2011). Episodic accretion may well occur. It is simply not capable of explaining the broad spread of optically visible PMS stars in the HRD.

We thank Kazu Omukai, Shu-ichiro Inutsuka, Christopher McKee, and Kevin Covey for fruitful discussions, and Dawn Peterson for providing us with her observational data. This work was initiated at the meeting "Multi-phase Interstellar Medium and Dynamics of Star Formation" held on 2010 February at Nagoya university. T.H. appreciates the support by Fellowship of the Japan Society for the Promotion of Science for

\footnotetext{
7 Baraffe \& Chabrier (2010) argue that episodic mass accretion also significantly influences the lithium depletion of low-mass stars. However, our present work suggests that variation of the initial models will be more significant than variation of the accretion histories for the problem of lithium depletion as well.

8 For example, the only cases BCG09 show where stars with $T_{\text {eff }}<3500 \mathrm{~K}$ lie near the 10 Myr non-accreting isochrone after $1 \mathrm{Myr}$ are their models A-C. However, they only use the initial conditions and accretion rates for models A-C to produce stars up to $0.2 M_{\odot}$. In comparison, all the models that they run to masses above $0.5 M_{\odot}$ have much higher starting entropies.
}

Research Abroad. M.R.K. acknowledges support from an Alfred P. Sloan Fellowship, from NSF through grants AST-0807739 and CAREER-0955300, and from NASA through Astrophysics Theory and Fundamental Physics grant NNX09AK31G and through a Spitzer Space Telescope Cycle 5 Theoretical Research Program grant. S.S.R.O. acknowledges support from NSF grant AST-0901055.

\section{REFERENCES}

Alexander, D. R., \& Ferguson, J. W. 1994, ApJ, 437, 879

Ardila, D. R., \& Basri, G. 2000, ApJ, 539, 834

Baraffe, I., \& Chabrier, G. 2010, A\&A, 521, A44

Baraffe, I., Chabrier, G., Allard, F., \& Hauschildt, P. H. 1998, A\&A, 337, 403

Baraffe, I., Chabrier, G., \& Gallardo, J. 2009, ApJ, 702, L27

Covey, K. R., Lada, C. J., Román-Zúñiga, C., Muench, A. A., Forbrich, J., \& Ascenso, J. 2010, ApJ, 722, 971

Da Rio, N., Gouliermis, D. A., \& Gennaro, M. 2010a, ApJ, 723, 166

Da Rio, N., Robberto, M., Soderblom, D. R., Panagia, N., Hillenbrand, L. A., Palla, F., \& Stassun, K. G. 2010b, ApJ, 722, 1092

D’Antona, F., \& Mazzitelli, I. 1994, ApJS, 90, 467

Dunham, M. M., Evans, N. J., Terebey, S., Dullemond, C. P., \& Young, C. H. 2010, ApJ, 710, 470

Elmegreen, B. G. 2000, ApJ, 530, 277

Enoch, M. L., Evans, N. J., Sargent, A. I., \& Glenn, J. 2009, ApJ, 692, 973

Evans, N. J., et al. 2009, ApJS, 181, 321

Gatti, T., Natta, A., Randich, S., Testi, L., \& Sacco, G. 2008, A\&A, 481, 423

Gatti, T., Testi, L., Natta, A., Randich, S., \& Muzerolle, J. 2006, A\&A, 460, 547

Gullbring, E., Calvet, N., Muzerolle, J., \& Hartmann, L. 2000, ApJ, 544, 927

Hartmann, L. 2001, AJ, 121, 1030

Hartmann, L. 2003, ApJ, 585, 398

Hartmann, L., Ballesteros-Paredes, J., \& Bergin, E. A. 2001, ApJ, 562, 852

Hartmann, L., Cassen, P., \& Kenyon, S. J. 1997, ApJ, 475, 770

Hayashi, C. 1961, PASJ, 13, 450

Hayashi, C., \& Nakano, T. 1963, Prog. Theor. Phys., 30, 460

Henyey, L. G., Lelevier, R., \& Levée, R. D. 1955, PASP, 67, 154

Hillenbrand, L. A. 2009, in IAU Symp. 258, The Ages of Stars, ed. E. E. Mamajek, D. R. Soderblom, \& R. F. G. Wyse (Cambridge: Cambridge Univ. Press), 81

Hosokawa, T., \& Omukai, K. 2009, ApJ, 691, 823

Hosokawa, T., Yorke, H. W., \& Omukai, K. 2010, ApJ, 721, 478

Iglesias, C. A., \& Rogers, F. J. 1996, ApJ, 464, 943

Kenyon, S. J., Hartmann, L. W., Strom, K. M., \& Strom, S. E. 1990, AJ, 99, 869

Kraus, A. L., \& Hillenbrand, L. A. 2009, ApJ, 704, 531

Krumholz, M. R., \& Tan, J. C. 2007, ApJ, 654, 304

Larson, R. B. 1969, MNRAS, 145, 271

Littlefair, S. P., Naylor, T., Mayne, N. J., Saunders, E. S., \& Jeffries, R. D. 2010, MNRAS, 403, 545

Machida, M. N., Inutsuka, S., \& Matsumoto, T. 2011, ApJ, 729, 42

Masunaga, H., \& Inutsuka, S. 2000, ApJ, 531, 350

Masunaga, H., Miyama, S. M., \& Inutsuka, S. 1998, ApJ, 495, 346

McKee, C. F., \& Offner, S. S. R. 2010, ApJ, 716, 167

Muzerolle, J., Luhman, K. L., Briceño, C., Hartmann, L., \& Calvet, N. 2005, ApJ, 625, 906

Offner, S. S. R., Klein, R. I., McKee, C. F., \& Krumholz, M. R. 2009, ApJ, 703, 131

Offner, S. S. R., \& McKee, C. F. 2011, ApJ, arXiv:1105.0671

Palla, F., \& Stahler, S. W. 1990, ApJ, 360, L47

Palla, F., \& Stahler, S. W. 1992, ApJ, 392, 667

Palla, F., \& Stahler, S. W. 1999, ApJ, 525, 772

Palla, F., \& Stahler, S. W. 2000, ApJ, 540, 255

Peterson, D. E., et al. 2008, ApJ, 685, 313

Prato, L., Greene, T. P., \& Simon, M. 2003, ApJ, 584, 853

Sestito, P., Palla, F., \& Randich, S. 2008, A\&A, 487, 965

Siess, L., Dufour, E., \& Forestini, M. 2000, A\&A, 358, 593

Siess, L., \& Forestini, M. 1996, A\&A, 308, 472

Siess, L., Forestini, M., \& Bertout, C. 1997, A\&A, 326, 1001

Slesnick, C. L., Hillenbrand, L. A., \& Carpenter, J. M. 2008, ApJ, 688, 377

Stahler, S. W. 1988, ApJ, 332, 804

Stahler, S. W., Shu, F. H., \& Taam, R. E. 1980, ApJ, 241, 637

Stassun, K. G., Mathieu, R. D., Cargile, P. A., Aarnio, A. N., Stempels, E., \& Geller, A. 2008, Nature, 453, 1079

Tan, J. C., Krumholz, M. R., \& McKee, C. F. 2006, ApJ, 641, L121

Vorobyov, E. I., \& Basu, S. 2005, ApJ, 633, L137

Winkler, K., \& Newman, M. J. 1980, ApJ, 236, 201 